\title{
Nonlinear Varying-Network Magnetic Circuit Analysis for Doubly Salient Permanent-Magnet Motors
}

\author{
Ming Cheng, K. T. Chau, Member, IEEE, C. C. Chan, Fellow, IEEE, E. Zhou, and X. Huang
}

\begin{abstract}
In this paper, a nonlinear varying-network magnetic circuit (VNMC) modeling method is developed for doubly salient permanent-magnet (DSPM) motors. The method is used to analyze the static characteristics of DSPM motors by specific permeance calculations, in which the interaction between the permanent-magnet field and the armature current field, as well as magnetic saturation, are taken into account. Two newly proposed DSPM motors (12/8-pole and 6/4-pole) are used as examples. There is good agreement between the results of the nonlinear VNMC modeling method and finite-element analysis. Moreover, the nonlinear VNMC method offers the advantage of calculating, effectively and efficiently, the static characteristics of DSPM motors having different dimensions, parameters, and conditions. Finally, the proposed method is verified by experimental testing of a 6/4-pole prototype.
\end{abstract}

Index Terms-Analytical model, doubly salient motors, magnetic circuits, permanent magnet motors, permeance calculation, static characteristics.

\section{INTRODUCTION}

D URING the past decade, switched reluctance (SR) motors have received much research attention. They offer the advantages of simple structure, fault tolerance, and mechanical robustness, leading to attractiveness for industrial and electric vehicle (EV) applications [1]. Nevertheless, their inherent deficiencies in efficiency and power density as compared to permanent-magnet (PM) brushless dc motors [2] have prompted research on the incorporation of PM's into the basic SR motor structure. Recently, a new class of motors, namely doubly salient permanent-magnet (DSPM) motors, has been introduced [3], [4]. A DSPM motor essentially adopts the same structure as a SR motor but with PM's placed in the stator or the rotor. Recent literature has already illustrated that DSPM motors are of high efficiency, high-power density, and simple structure [3]-[6].

Static characteristics are the basis of design, analysis, and control of various motors, and therefore are of the first importance for DSPM motors. Although these motors possess simplicity in construction, they do not offer any simplicity in anal-

Manuscript received May 10, 1999; revised September 17, 1999. This work was partially supported by a grant from the NSFC Project 59507001 China, and a grant from the RGC Project HKU 7128/99E, Hong Kong, China.

M. Cheng, K.T. Chau, and C.C. Chan are with the Department of Electrical and Electronic Engineering, University of Hong Kong, Hong Kong, China (e-mail: mcheng@eee.hku.hk; ktchau@eee.hku.hk; ccchan@eee.hku.hk).

E. Zhou and X. Huang are with the Department of Electrical Engineering, Southeast University, Nanjing 210096, China (e-mail: ezhou@ seu.edu.cn).

Publisher Item Identifier S 0018-9464(00)00477-5. ysis. Because of the heavy magnetic saturation of pole tips and the fringe effect of poles and slots, as well as the cross coupling between the PM flux and the armature current flux, the analysis of DSPM motors presents great difficulty. By using finite-element analysis (FEA), the magnetic field distribution and hence the static characteristics of DSPM motors can be calculated accurately [7]. However, FEA is inconvenient and timeconsuming for design and analysis of DSPM motors in which different geometric dimensions need to be considered.

The objective of this paper is to propose an effective and efficient method, with reasonable accuracy, for the design and analysis of DSPM motors. A nonlinear varying-network magnetic circuit (VNMC) model for DSPM motors leading to formulas for the calculation of the airgap permeances will be presented. Moreover, nonideality in airgap and pole permeances will be taken into account to improve the calculation accuracy. The proposed method will be applied to two new DSPM motors, namely 12/8-pole and 6/4-pole, for evaluation. Experimental results on a 6/4-pole prototype will be presented for verification of the calculations.

\section{NONLINEAR VNMC MODEL}

Fig. 1 shows the configuration of two DSPM motors, namely 6/4-pole and 12/8-pole. Due to symmetry, the 12/8-pole DSPM motor can be considered as two 6/4-pole motors. Hence the modeling is needed only for the 6/4-pole motor.

Fig. 2 shows the nonlinear VNMC model of the 6/4-pole DSPM motor at two typical rotor positions, namely the minimum flux position $\left(\theta=0^{\circ}\right)$ and the maximum flux position $\left(\theta=45^{\circ}\right)$ of phase $\mathrm{A}$, where $\theta$ is defined as the angle between the stator pole central line and the rotor slot central line as shown in Fig. 1(a). In the model, $p_{P S}, p_{Y S}\left(p_{Y S}^{\prime}\right), p_{P R}$, and $p_{Y R}$ are the pole and yoke permeances of the stator and rotor, respectively, which vary with the nonlinear saturation in the corresponding magnetic paths, while $p_{P Y L}, p_{P L}, p_{P \mathrm{M}}, p_{P M L}$, and $p_{A}$ are the permeances of stator pole-to-yoke leakage flux, stator pole-to-pole leakage flux, PM flux, PM leakage flux, and airgap flux, respectively, which are of constant permeability. The PM magnetizing force is represented by an equivalent MMF $F_{\mathrm{PM}}$. In order to take into account $p_{P Y}$, the armature MMF supplied by phase A winding, the stator pole permeance, and the stator yoke permeance are represented by two $F_{A}$, two $p_{P S}$, and two $p_{Y S}$, respectively. On the other hand, $p_{Y S}^{\prime}$ stands for the stator yoke permeance when there is no need to consider $p_{P Y L}$. It should be noted that both the value and the number of $p_{A}$ in the 


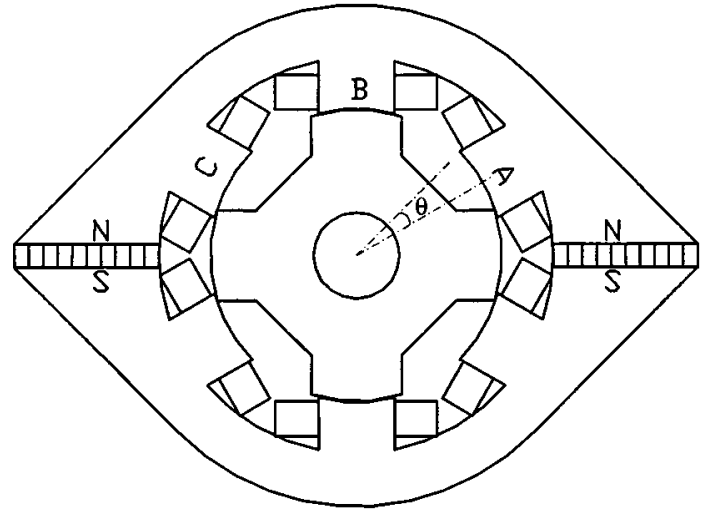

(a)

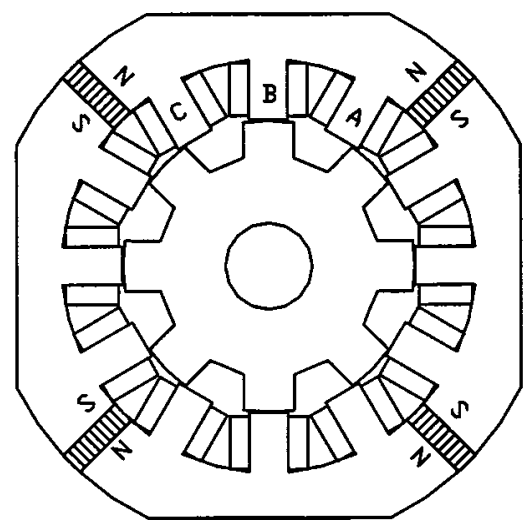

(b)

Fig. 1. Configuration of two DSPM motors: (a) 6/4-pole and (b) 12/8-pole.

model change with the rotor position as shown in the two circuits in Fig. 2 (one is of $8 p_{A}$ branches whereas the other is of $10 p_{A}$ branches), hence the so-called nonlinear VNMC model.

\section{SPecific Permeance Calculation}

The analysis of the airgap specific permeance (permeance per axial length) at various rotor positions is based on a permeance calculation method using flux tubes with boundaries determined by semicircular and straight segments [8]-[10]. For simplification, the following assumptions are made for initial characterization of the airgap permeance.

- The permeability of ferromagnetic materials is infinite and hence the pole surfaces of both stator and rotor are individually equipotential.

- Magnetic force lines are perpendicular to the iron surfaces.

- The magnetic field distributes evenly along the axial direction.

According to the relative position of the rotor with respect to the stator, there are eight possible regions as defined by

$$
\begin{cases}\text { Region 1: } & 0 \leq \alpha<\alpha_{1} \\ \text { Region 2: } & \alpha_{1} \leq \alpha<\alpha_{2} \\ \cdots & \\ \text { Region 8: } & \alpha_{7} \leq \alpha<\alpha_{8}\end{cases}
$$

where $\alpha$ is defined as the angle between the central lines of the stator and rotor poles, and $\alpha_{1}-\alpha_{8}$ depend on the structural dimensions of the motor. Those rotor positions corresponding

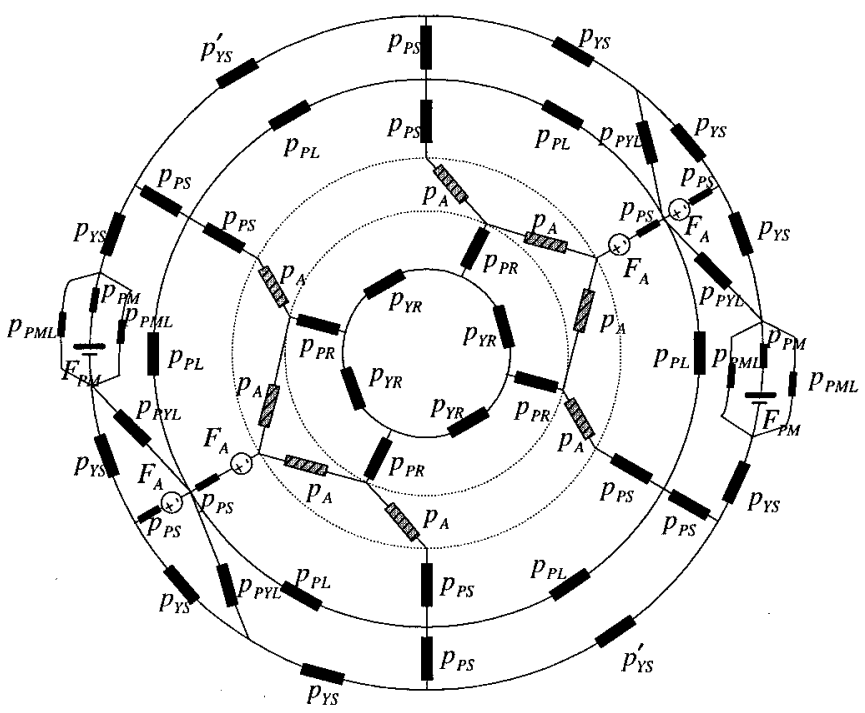

(a)

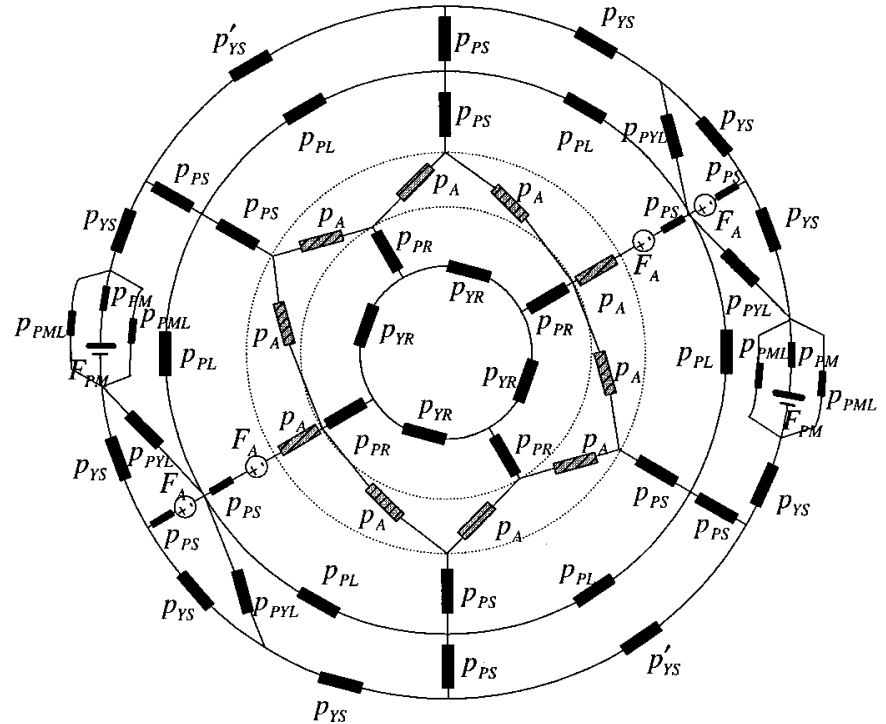

(b)

Fig. 2. Nonlinear VNMC model of 6/4-pole DSPM motor: (a) $\theta=0^{\circ}$ and (b) $\theta=45^{\circ}$.

to various regions and $\alpha=\alpha_{1}-\alpha_{8}$ are shown in Figs. 3 and 4, respectively, in which $\tau_{s}$ is the stator pole pitch, $\tau_{r}$ is the rotor pole pitch, $\beta_{s}$ is the stator pole arc, and $\beta_{r}$ is the rotor pole arc. From Fig. 4, $\alpha_{1}-\alpha_{8}$ can be expressed in terms of $\tau_{s}, \tau_{r}, \beta_{s}$, and $\beta_{r}$. For example, $\alpha_{1}$ is given by

$$
\alpha_{1}=\frac{1}{2}\left(\beta_{r}-\beta_{s}\right) \text {. }
$$

As shown in Fig. 3, all regions are divided into several sections, namely $a-c, a-d$, or $a-e$, in which the magnetic parameters within each section are considered to be constant. For example, Region 1 is divided into five sections that can be expressed as

$$
\begin{aligned}
a & =\left[\frac{1}{2}\left(\tau_{s}-\beta_{r}\right)+\alpha\right] r_{s i} \\
b & =\left(\alpha_{1}-\alpha\right) r_{s i} \\
c & =\beta_{s} r_{s i} \\
d & =\left(\alpha_{1}+\alpha\right) r_{s i} \\
e & =\frac{1}{2}\left(\tau_{s}-\beta_{s}\right) r_{s i}-d
\end{aligned}
$$




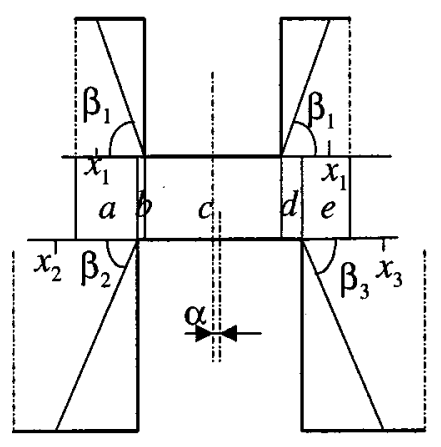

(a)

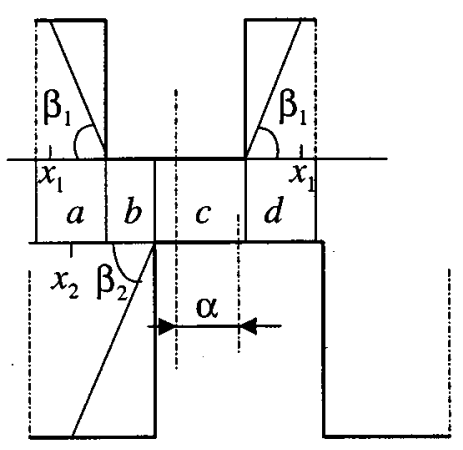

(c)

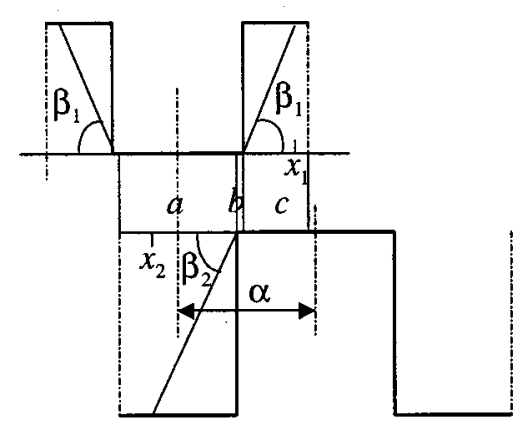

(e)

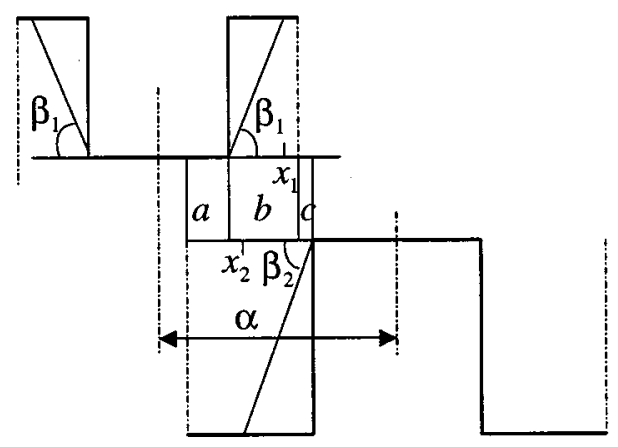

(g)

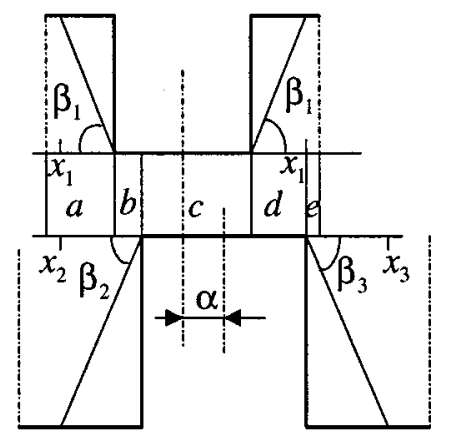

(b)

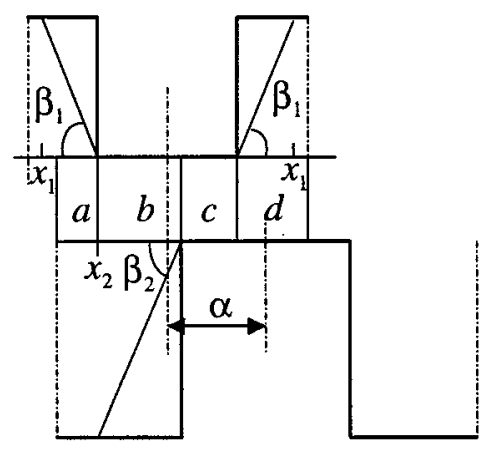

(d)

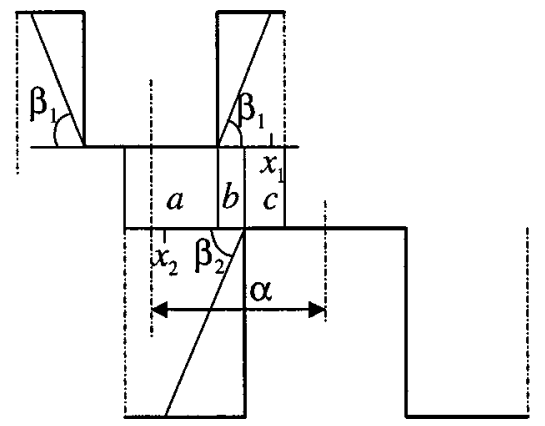

(f)

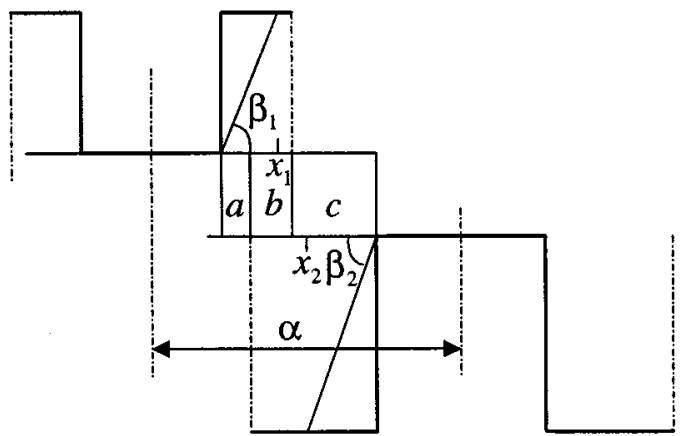

(h)

Fig. 3. Rotor positions at different regions. (a) Region one. (b) Region two. (c) Region three. (d) Region four. (e) Region five. (f) Region six. (g) Region seven. (h) Region eight.

where $r_{s i}$ is the stator inner radius. Moreover, in order to analytically describe the flux lines by semicircular and straight segments [8]-[10], the edges of the stator pole as well as the left and right edges of the rotor pole are inclined by the angles of $\beta_{1}, \beta_{2}$, and $\beta_{3}$, respectively, instead of $\pi / 2$, as shown in Fig. 3 . As a rule of thumb, these angles can be represented by empirical values [8], or expressed by analytical formulas [11]

$$
\begin{aligned}
& \beta_{1}=\pi / 2-\beta_{s} / 2 \\
& \beta_{2}=\pi / 2-\beta_{r} / 2 \\
& \beta_{3}=\pi / 2-\beta_{r} / 2 .
\end{aligned}
$$




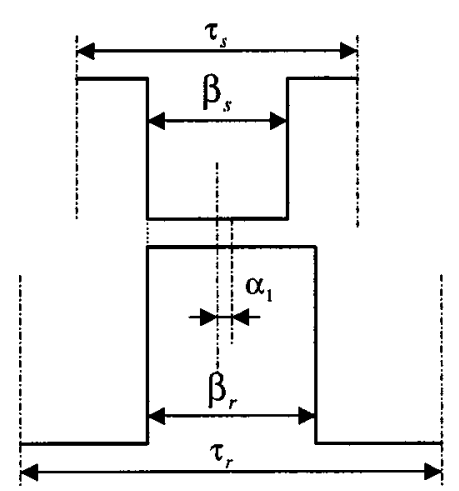

(a)

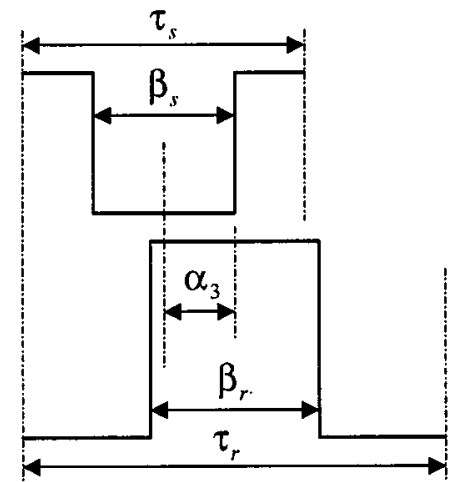

(c)

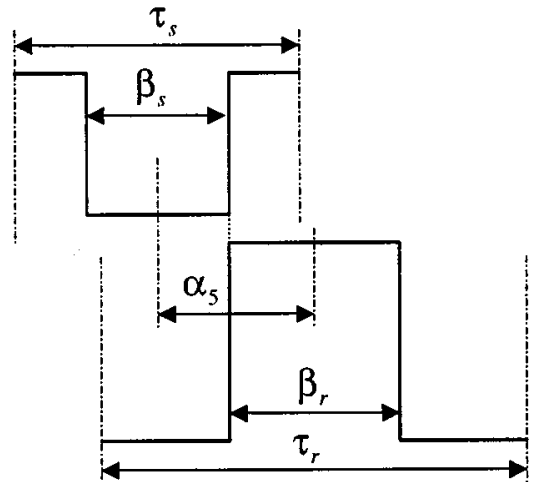

(e)

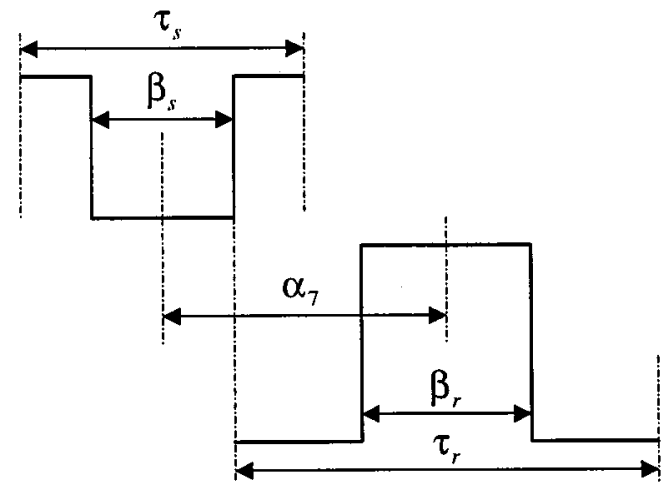

(g)

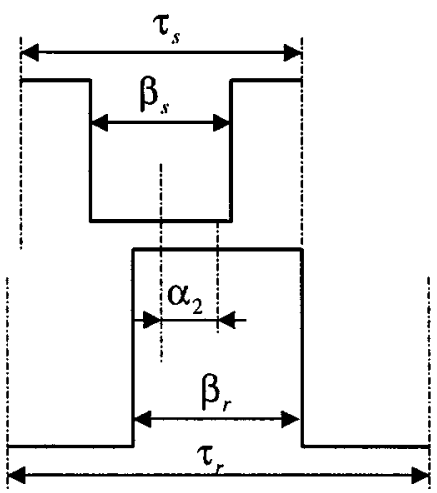

(b)

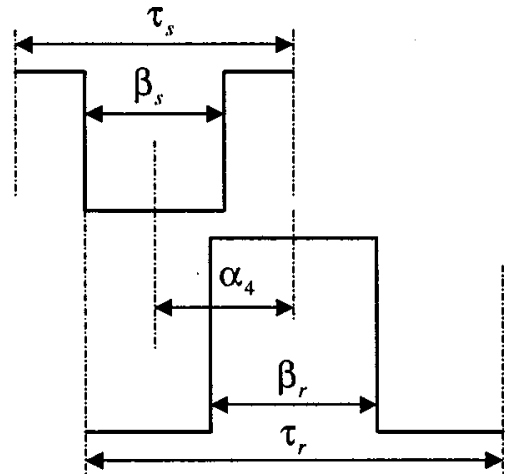

(bd)

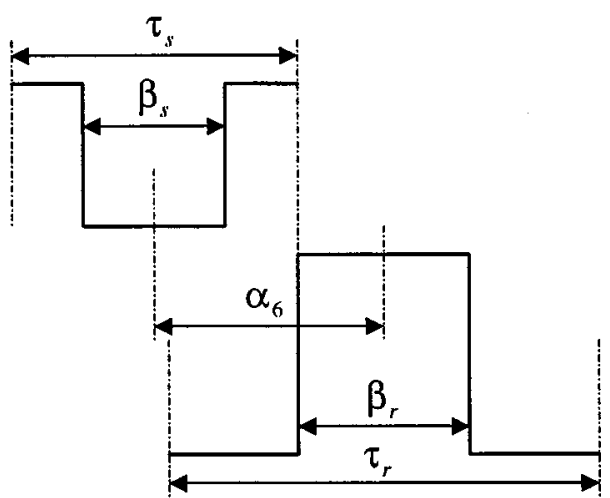

(f)

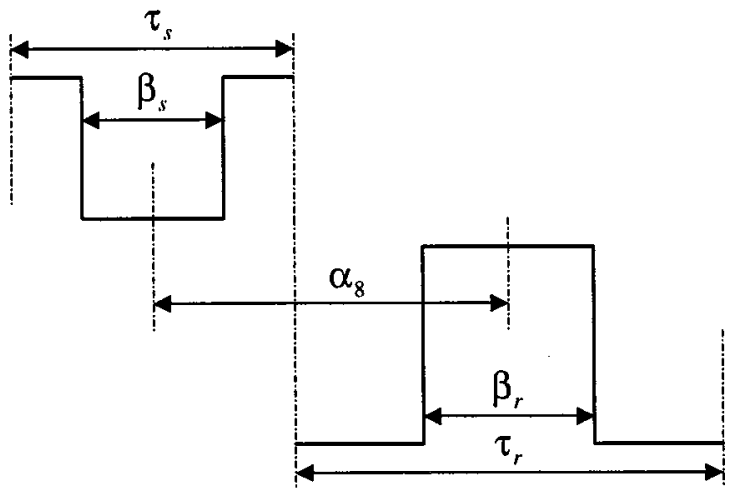

(h)

Fig. 4. Rotor positions at different $\alpha$. (a) $\alpha=\alpha_{1}$. (b) $\alpha=\alpha_{2}$. (c) $\alpha=\alpha_{3}$. (d) $\alpha=\alpha_{4}$. (e) $\alpha=\alpha_{5}$. (f) $\alpha=\alpha_{6}$. (g) $\alpha=\alpha_{7}$. (h) $\alpha=\alpha_{8}$. 
Corresponding to $\beta_{1}, \beta_{2}$, and $\beta_{3}$, the interconnection points between those semicircular and straight segments are given by

$$
\left\{\begin{array}{l}
x_{1}=h_{s} / \tan \beta_{1} \\
x_{2}=h_{r} / \tan \beta_{2} \\
x_{3}=h_{r} / \tan \beta_{3}
\end{array}\right.
$$

where $h_{s}$ and $h_{r}$ are the stator and rotor pole heights, respectively.

The airgap specific permeance of a flux tube can be calculated from the following formula:

$$
p=\mu_{0} A_{m} / l_{m}
$$

where

$A_{m} \quad$ width of the flux tube;

$l_{m} \quad$ length of flux path between equipotential surfaces;

$\mu_{0} \quad$ permeability of free space.

In the following, Region 1 will be used to illustrate the formulation of the airgap specific permeance. In Section $a$, the magnetic flux path consists of two semicircular and one straight segment while the corresponding length varies with distance $x$ from the left edge of rotor pole. Thus, by using (12), the airgap specific permeance in section $a$ is given as

$$
\begin{aligned}
p_{A a} & =\int_{0}^{a} \frac{\mu_{0} d x}{g_{0}+\beta_{1}(x+b)+\beta_{2} x} \\
& =\frac{\mu_{0}}{\beta_{1}+\beta_{2}} \ln \left[1+\frac{\left(\beta_{1}+\beta_{2}\right) a}{g_{0}+\beta_{1} b}\right]
\end{aligned}
$$

where $g_{0}$ is the airgap length, provided that the span of $\beta_{2}$ is not less than the length of section $a$

$$
x_{2} \geq a
$$

Otherwise, it should be expressed as

$$
\begin{aligned}
p_{A a}= & \int_{0}^{x_{2}} \frac{\mu_{0} d x}{g_{0}+\beta_{2} x+\beta_{1}(x+b)} \\
& +\int_{x_{2}}^{a} \frac{\mu_{0} d x}{g_{0}+h_{r}+\beta_{1}(x+b)} \\
= & \frac{\mu_{0}}{\beta_{1}+\beta_{2}} \ln \left[1+\frac{\left(\beta_{1}+\beta_{2}\right) x_{2}}{g_{0}+\beta_{1} b}\right] \\
& +\frac{\mu_{0}}{\beta_{1}} \ln \left[1+\frac{\beta_{1}\left(a-x_{2}\right)}{g_{0}+h_{r}+\beta_{1}\left(x_{2}+b\right)}\right] .
\end{aligned}
$$

Since the magnetic flux path in section $b$ consists of one semicircular and one straight segment, the corresponding specific permeance is given by

$$
p_{A b}=\int_{0}^{b} \frac{\mu_{0} d x}{g_{0}+\beta_{1} x}=\frac{\mu_{0}}{\beta_{1}} \ln \left[1+\frac{\beta_{1} b}{g_{0}}\right] .
$$

In section $c$, since the magnetic flux path is kept constant at $g_{0}$, the corresponding specific permeance is simply written as

$$
p_{A c}=\frac{\mu_{0} c}{g_{0}} .
$$

Similar to (16), the specific permeance in section $d$ is given by

$$
p_{A d}=\int_{0}^{d} \frac{\mu_{0} d x}{g_{0}+\beta_{1} x}=\frac{\mu_{0}}{\beta_{1}} \ln \left[1+\frac{\beta_{1} d}{g_{0}}\right] .
$$

Similar to (13), the specific permeance in section $e$ is given by

$$
\begin{aligned}
p_{A e} & =\int_{0}^{e} \frac{\mu_{0} d x}{g_{0}+\beta_{1}(x+d)+\beta_{3} x} \\
& =\frac{\mu_{0}}{\beta_{1}+\beta_{3}} \ln \left[1+\frac{\left(\beta_{1}+\beta_{3}\right) e}{g_{0}+\beta_{1} d}\right]
\end{aligned}
$$

provided that the span of $\beta_{3}$ is not less than the length of section $e$

$$
x_{3} \geq e
$$

Otherwise, it is given by

$$
\begin{aligned}
p_{A e}= & \int_{0}^{x_{3}} \frac{\mu_{0} d x}{g_{0}+\beta_{1}(x+d)+\beta_{3} x} \\
& +\int_{x_{3}}^{e} \frac{\mu_{0} d x}{g_{0}+h_{r}+\beta_{1}(x+d)} \\
= & \frac{\mu_{0}}{\beta_{1}+\beta_{3}} \ln \left[1+\frac{\left(\beta_{1}+\beta_{3}\right) x_{3}}{g_{0}+\beta_{1} d}\right] \\
& +\frac{\mu_{0}}{\beta_{1}} \ln \left[1+\frac{\beta_{1}\left(e-x_{3}\right)}{g_{0}+h_{r}+\beta_{1}\left(d+x_{3}\right)}\right] .
\end{aligned}
$$

Therefore, making use of (13)-(21), the total airgap specific permeance is formulated as

$$
p_{A}(\alpha)=\sum_{i=a}^{e} p_{A i}
$$

For the other regions, the total airgap specific permeances can be derived in a similar way. The corresponding formulas are omitted in this paper for the sake of conciseness. On the other hand, the specific permeance of iron in the magnetic circuit is similarly determined by using (12), but with a nonconstant permeability $\mu$ that needs to be determined by iterative calculation.

\section{CORRECTION FOR NONIDEALITY}

\section{A. Nonideal Airgap Permeance}

Due to saturation in the pole, especially in the pole tip, the airgap specific permeance derived under the above ideal conditions will be larger than its actual value. To improve the accuracy, a correction term is introduced which is defined by

$$
k_{c}=\frac{p_{A}(\alpha)}{p_{A}^{\prime}(\alpha)}=1+\frac{c_{1}}{1+c_{2} \alpha}
$$

where $p_{A}^{\prime}(\alpha)$ is the modified airgap specific permeance. Coefficients $c_{1}$ and $c_{2}$ are specified based on FEA. Once the airgap specific permeances at two typical positions, namely $\alpha=0^{\circ}$ and $\alpha=45^{\circ}$, are obtained by FEA, $c_{1}$ is determined by comparing the airgap permeances obtained by FEA and VNMC at $\alpha=0^{\circ}$, and then $c_{2}$ is calculated by comparing those airgap permeances at $\alpha=45^{\circ}$.

\section{B. Nonideal Pole Permeance}

Fig. 5 shows the local magnetic field distribution when the stator and rotor poles are partly overlapping. There is uneven magnetic field distribution in the pole area near the airgap and significant local saturation in the pole tips. To take into account this effect, the lower half of the stator pole is divided into two 


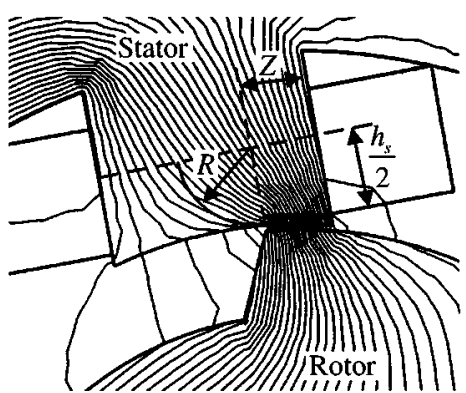

Fig. 5. Magnetic field distribution during partly overlapping of poles.

portions in such a way that the length of the magnetic flux lines in the nonoverlapped portion can be approximately represented by an arc with radius $R$ while the effective width of this portion is considered as one half of its physical value. Thus, the pole permeance is composed of two components, due to the overlapped and nonoverlapped portions, as given by

$$
\begin{gathered}
p_{P}=p_{P 1}+p_{P 2} \\
p_{p 1}=\mu_{p 1} \frac{z}{h_{s} / 2} \\
p_{p 2}=\mu_{p 2} \frac{\left(b_{s}-z\right) / 2}{\pi R / 2}=\mu_{p 2} \frac{b_{s}-z}{\pi R}
\end{gathered}
$$

where

$b_{s} \quad$ width of stator pole;

$z \quad$ width of the corresponding overlapped portion;

$\mu_{p 1}$ and $\mu_{p 2} \quad$ permeability of the overlapped and nonoverlapped portions, respectively.

\section{Establishment of the Magnetic CiRCUIT EQuations}

As mentioned above, the number of branches of the equivalent magnetic circuit in Fig. 2 varies with the rotor position, while the number of nodes in the circuit is fixed. Hence, nodal analysis is employed to solve the magnetic circuit. In the equivalent circuit, any branch can be represented by two possible magnetic branches as shown in Fig. 6, in which $k_{i 1}$ and $k_{i 2}$ represent the nodes connected by branch $i, F_{m}\left(k_{i 1}\right)$ and $F_{m}\left(k_{i 2}\right)$ are the node magnetic potentials, $F_{m 0}(i)$ is the branch magnetomotive source, $\phi_{0}(i)$ is the branch magnetic flux source, $G_{m}(i)$ is the branch permeance, $\phi(i)$ is the branch magnetic flux flow, and $\Delta F_{m}(i)$ is the branch magnetic potential difference. These parameters have the following relationship:

$$
\left\{\begin{array}{c}
\Delta F_{m}(i)=F_{m}\left(k_{i 1}\right)-F_{m}\left(k_{i 2}\right) \\
\phi(i)=G_{m}(i) \Delta F_{m}(i)+\phi_{0}(i) \\
\phi_{0}(i)=G_{m}(i) F_{m 0}(i)
\end{array} .\right.
$$

Therefore, any branch in the magnetic circuit can be defined by five variables, namely $i, k_{i 1}, k_{i 2}, G_{m}(i)$, and $F_{m 0}(i)$ or $\phi_{0}(i)$.

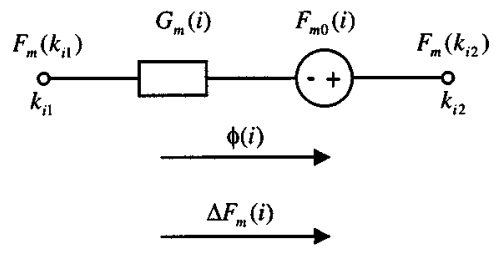

(a)

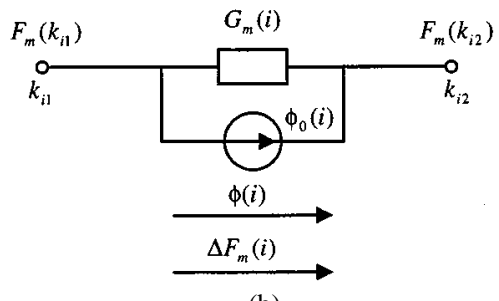

(b)

Fig. 6. Possible magnetic branches: (a) magnetomotive source and (b) magnetic flux source.

The nonlinear equation describing the relationship of these magnetic variables can be established as follows:

$\left[\begin{array}{cccc}G(1,1) & G(1,2) & \cdots & G(1, n) \\ G(2,1) & G(2,2) & \cdots & G(2, n) \\ \vdots & \vdots & \vdots & \vdots \\ G(n, 1) & G(n, 2) & \cdots & G(n, n)\end{array}\right]\left[\begin{array}{c}F_{m}(1) \\ F_{m}(2) \\ \vdots \\ F_{m}(n)\end{array}\right]=\left[\begin{array}{c}\phi_{s}(1) \\ \phi_{s}(2) \\ \vdots \\ \phi_{s}(n)\end{array}\right]$

where

$n$

\section{dependent on these nodes)}

$G\left(n_{1}, n_{2}\right) \quad$ for $n_{1}, n_{2}=1 \cdots n$ is the node permeance;

$F_{m}\left(n_{1}\right) \quad$ for $n_{1}=1 \cdots n$ is the node magnetic vector potential;

$\phi_{s}\left(n_{1}\right) \quad$ for $n_{1}=1 \cdots n$ is the node magnetic flux source.

In short form, it is rewritten as

$$
\boldsymbol{G}\left(\mu_{i}\right) \mathbf{F}_{m}=\Phi_{s}\left(\mu_{i}\right)
$$

where

$\mu_{i} \quad$ permeability of branch $i$;

$\mathbf{G}\left(\mu_{i}\right)$ node permeance matrix;

$\mathbf{F}_{m} \quad$ vector of the node magnetic potential;

$\Phi_{s}\left(\mu_{i}\right)$ vector of the node magnetic flux source.

Considering the $k$ th node of (28), the coefficient of the kth node magnetic potential is the sum of those branch permeances connected to the node $k$, while the coefficients of the other node magnetic potentials are the negatives of the branch permeances between those nodes and the node $k$. On the other hand, the $k$ th node magnetic flux source corresponds to the net magnetic flux flowing into the node $k$. Hence, it yields the following computational procedure:

$$
\left\{\begin{aligned}
G\left(k_{i 1}, k_{i 1}\right) \leftarrow G\left(k_{i 1}, k_{i 1}\right)+G_{m}(i) \\
G\left(k_{i 1}, k_{i 2}\right) \leftarrow G\left(k_{i 1}, k_{i 2}\right)-G_{m}(i) \\
G\left(k_{i 2}, k_{i 1}\right)=G\left(k_{i 1}, k_{i 2}\right) \\
G\left(k_{i 2}, k_{i 2}\right) \leftarrow G\left(k_{i 2}, k_{i 2}\right)+G_{m}(i) \\
\phi_{s}\left(k_{i 1}\right) \leftarrow \phi_{s}\left(k_{i 1}\right)-\phi_{0}(i) \\
\phi_{s}\left(k_{i 2}\right) \leftarrow \phi_{s}\left(k_{i 2}\right)+\phi_{0}(i) \\
i=1,2, \ldots, N_{b}
\end{aligned}\right.
$$

where $N_{b}$ is the total number of branches in the magnetic circuit. 


\section{STATIC ChARACTERISTICS}

By rotating the rotor of the 6/4-pole motor from $0^{\circ}$ to $90^{\circ}$ ( $0^{\circ}$ to $45^{\circ}$ for the $12 / 8$-pole motor) step by step, the airgap specific permeance between the stator and rotor poles can be calculated. For example, in section $a$ of region one, the permeance can be obtained from (13) to (22). If this permeance is nonzero, there will be an airgap permeance branch between the corresponding stator and rotor poles. Having determined the structure of the network circuit, the node permeance matrix $\mathbf{G}\left(\mu_{i}\right)$ and the node magnetic flux source vector $\Phi_{s}\left(\mu_{i}\right)$ can be formed by using (30). Hence, the magnetic circuit equation given by (29) can be established. Since both $\mathbf{G}\left(\mu_{i}\right)$ and $\Phi_{s}\left(\mu_{i}\right)$ are functions of permeability, iterative calculation is required to solve the equation.

In the $k$ th iteration, (29) can be rewritten as

$$
\mathbf{G}\left(\mu_{i}^{(k-1)}\right) \mathbf{F}_{m}^{(k)}=\Phi_{s}\left(\mu_{i}^{(k-1)}\right) \text {. }
$$

Once $\mathbf{F}_{m}^{(k)}$ is solved, $\phi(i)^{(k)}\left(i=1,2, \ldots, N_{b}\right)$ can be obtained by (27). Then, the relevant magnetic flux density $B_{i}^{(k)}$ is given by

$$
B_{i}^{(k)}=\phi(i)^{(k)} / A_{i}
$$

where $A_{i}$ is the area of branch $i$. From the magnetization curve of the core material, the corresponding permeability $\hat{\mu}_{i}^{(k)}$ is obtained as

$$
\hat{\mu}_{i}^{(k)}=B_{i}^{(k)} / H_{i}^{(k)}
$$

where $H_{i}^{(k)}$ is the magnetic field intensity corresponding to $B_{i}^{(k)}$. Thus, the permeability for the $(k+1)$ th iteration is determined by the following expression:

$$
\begin{gathered}
\mu_{i}^{(k)}=\mu_{i}^{(k-1)}+p_{i}^{(k)}\left(\hat{\mu}_{i}^{(k)}-\mu_{i}^{(k-1)}\right) \\
p_{i}^{(k)}=\min \left\{1.0,0.01+\frac{c_{d}}{c_{d}+\left|\hat{\mu}_{i}^{(k)}-\mu_{i}^{(k-1)}\right| / \mu_{i}^{(k-1)}}\right\}
\end{gathered}
$$

where

$p_{i}^{(k)} \quad$ damping factor, and

$c_{d} \quad$ damping constant that is selected to be 0.7 .

The above iterative process is ended when the following criterion is satisfied

$$
\left|\frac{\hat{\mu}_{i}^{(k)}-\mu_{i}^{(k-1)}}{\mu_{i}^{(k-1)}}\right| \leq \varepsilon
$$

where $\varepsilon$ is the end factor determined by the accuracy requirement.

Based on the solution of (29), the static characteristics of the DSPM motor are readily obtained. Fig. 7 shows the PM flux linkages of the two DSPM prototypes, namely 6/4-pole and 12/8-pole, at no-load determined by the proposed VNMC approach. Similar results obtained from FEA are also plotted in Fig. 7. It may be noted that there is a good agreement between them.

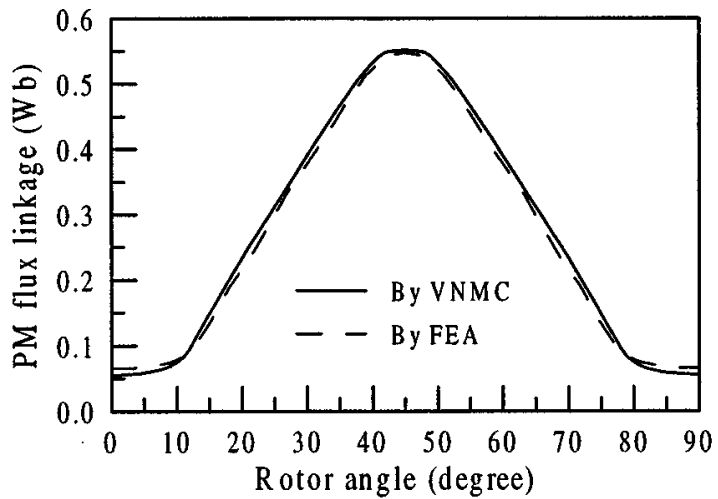

(a)

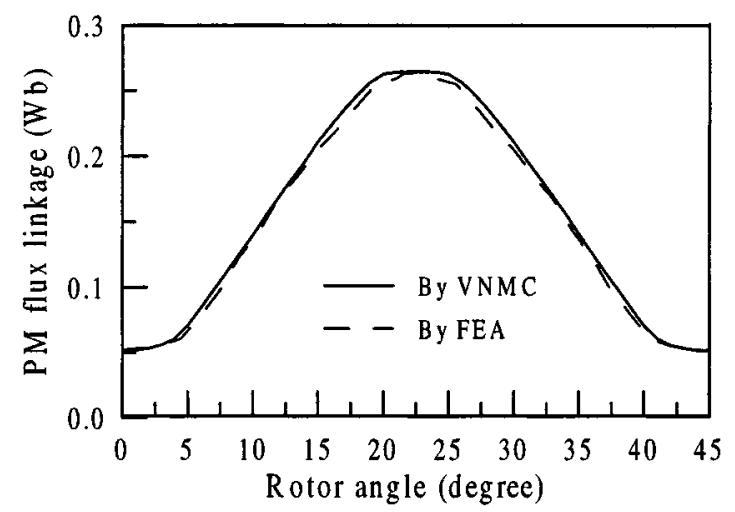

(b)

Fig. 7. PM flux linkages calculated by VNMC and FEA: (a) 6/4-pole and (b) 12/8-pole.

From the PM flux linkage versus rotor angle as shown in Fig. 7, the EMF can be deduced by

$$
\mathrm{EMF}=\frac{d \psi_{m}}{d t}=\frac{d \psi_{m}}{d \theta} \omega
$$

where

$\psi_{m} \quad$ PM flux linkage, and

$\omega \quad$ angular velocity of the rotor.

Based on $\psi_{m}$ resulting from the proposed VNMC approach, the theoretical EMF waveform for the 6/4-pole DSPM motor at $1500 \mathrm{rpm}$ is shown in Fig. 8(a). Compared with the measured waveform shown in Fig. 8(b), the waveforms resemble one another although with some discrepancies. These discrepancies should be due to the imperfection in manufacture.

In the calculation of inductances, the cross coupling between PM flux and armature flux is considered. In the presence of both PM field and armature current field, the overall flux linkage $\psi$ is given by:

$$
\psi=\psi_{m}+L i_{a}
$$

where $i_{a}$ is the armature current per phase. Thus, the inductance can be expressed as

$$
L=\frac{\psi-\psi_{m}}{i_{a}} .
$$

Fig. 9 illustrates these inductances versus rotor angle under different load conditions, in which "PM $+1 \mathrm{~A}$ " and "PM - 1A" denote the strengthening and weakening actions of the arma- 


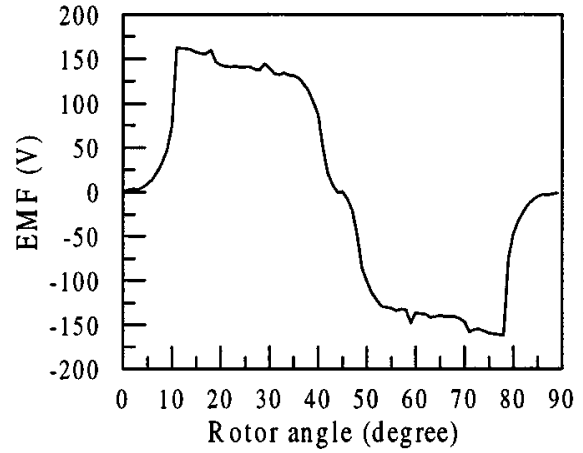

(a)

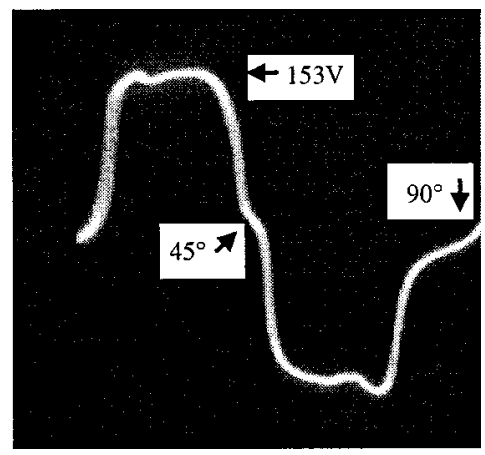

(b)

Fig. 8. EMF waveforms of 6/4-pole motor at $1500 \mathrm{rpm}$. (a) Theoretical. (b) Measured.

ture current field of $i_{a}=1$ A to the PM field, respectively, while Fig. 10 illustrates the inductances versus rotor angle under "PM $+2 \mathrm{~A}$ " and "PM $-2 \mathrm{~A}$." It is obvious that the inductance of the motor is affected significantly by the cross coupling between PM field and armature current field. Tables I and II give comparisons on the inductance values of the 6/4-pole prototype at two typical rotor positions when $i_{a}=1.4 \mathrm{~A}$ and $2.5 \mathrm{~A}$, respectively, resulting from the VNMC approach, FEA, and experimental measurement. It may be seen that the FEA inductances closely match with the measured values while the VNMC inductances exhibit slightly higher discrepancies. These discrepancies are reasonable and expected because the proposed VNMC approach takes advantage of analytical modeling to rapidly determine the static characteristics of DSPM motors with different dimensions and excitation conditions. The degradation of accuracy is tolerable in view of the time saving.

It may be noted that the discrepancy between the VNMC approach and FEA as shown in Fig. 9(a) is relatively larger than that in Fig. 9(b). It is due to the fact that the correction terms for local saturation in the pole tips given by (24)-(26) are to improve the model accuracy rather than to eliminate the effect of local saturation. Since the pole width of a 6/4-pole motor is larger than that of a 12/8-pole motor, the effect of local saturation in the pole tips and hence the discrepancy in a 6/4-pole motor are higher than that in a 12/8-pole motor. The difference in the discrepancies shown in Fig. 10(a) and (b) can be explained in a similar way.

In order to show the rapidity of the proposed approach, Table III gives a comparison of computation times between VNMC and FEA based on a Pentium PC. It is obvious that, even ignoring the preprocessing time for FEA, the proposed

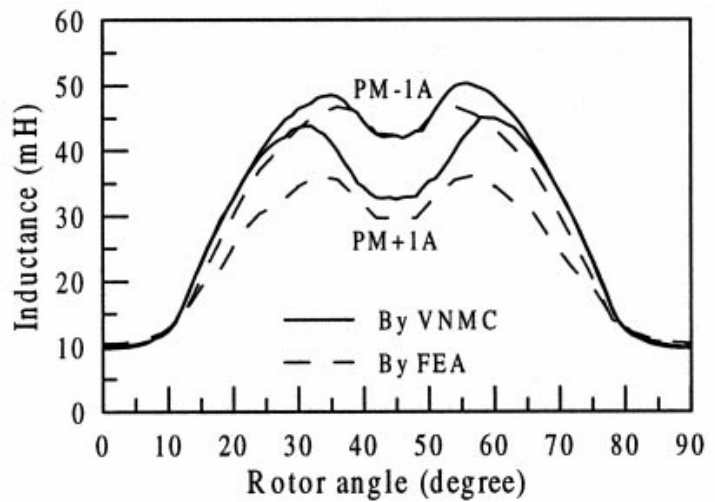

(a)

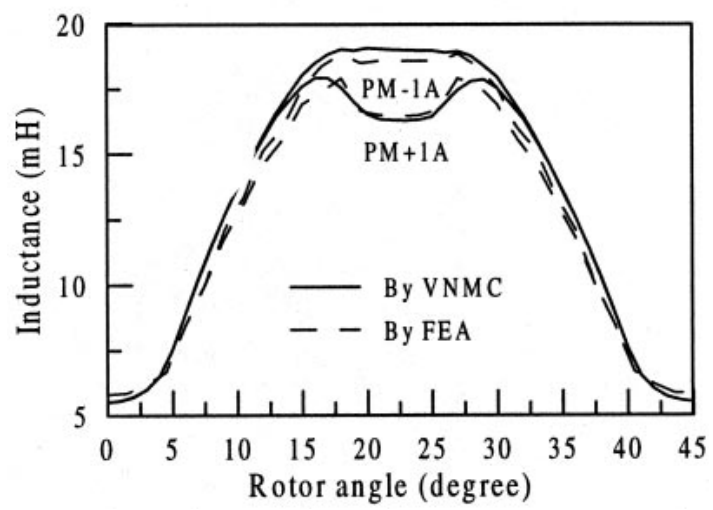

(b)

Fig. 9. Inductances at armature current of 1 A. (a) 6/4-pole. (b) 12/8-pole.

TABLE I

INDUCTANCES OF 6/4-POLE MOTOR AT

$i_{a}=1.4 \mathrm{~A}$

\begin{tabular}{c|ccc|ccc|c}
\hline$\theta$ & \multicolumn{3}{|c|}{ VNMC Inductance $(\mathrm{mH})$} & \multicolumn{3}{|c|}{ FEA Inductance $(\mathrm{mH})$} & \multirow{2}{*}{$\begin{array}{c}\text { Measured } \\
\text { Inductance }(\mathrm{mH})\end{array}$} \\
\cline { 2 - 7 }${ }^{\circ}$ & $\mathrm{PM}+i_{a}$ & PM- $i_{a}$ & Average & PM $+i_{a}$ & PM- $i_{a}$ & Average & \\
\hline 15 & 23.00 & 22.63 & 22.81 & 19.08 & 20.44 & 19.76 & 20.1 \\
45 & 31.77 & 44.74 & 38.26 & 29.55 & 42.40 & 35.98 & 34.2 \\
\hline
\end{tabular}

TABLE II

INDUCTANCES OF 6/4-POLE MOTOR AT $i_{a}=2.5 \mathrm{~A}$

\begin{tabular}{c|ccc|ccc|c}
\hline$\theta$ & \multicolumn{3}{|c|}{ VNMC Inductance $(\mathrm{mH})$} & \multicolumn{3}{|c|}{ FEA Inductance $(\mathrm{mH})$} & \multirow{2}{*}{$\begin{array}{c}\text { Measured } \\
\text { Inductance }(\mathrm{mH})\end{array}$} \\
\cline { 2 - 6 }$\left(^{\circ}\right)$ & $\mathrm{PM}+i_{\alpha}$ & $\mathrm{PM}-i_{\alpha}$ & Average & PM $+i_{\alpha}$ & PM- $i_{\alpha}$ & Average & \\
\hline 15 & 22.14 & 21.83 & 21.99 & 18.24 & 20.49 & 19.37 & 20.5 \\
45 & 30.10 & 50.18 & 40.14 & 26.83 & 47.14 & 36.99 & 39.8 \\
\hline
\end{tabular}

VNMC approach is much faster than FEA. Actually, it is the expected advantage due to analytical modeling.

Based on the resulting PM flux linkage and inductance, the static torque characteristics for one-phase-on condition can be deduced by

$$
T_{e}=\frac{1}{2} i_{a}^{2} \frac{d L}{d \theta}+i_{a} \frac{d \psi_{m}}{d \theta}=T_{r}+T_{p m}
$$

where

$T_{e}$

$T_{r}=$

$(1 / 2) i_{a}^{2}(d L / d \theta)$

$T_{p m}=$

$i_{a}\left(d \psi_{m} / d \theta\right)$ total electromagnetic torque; reluctance torque due to the variation of inductance; and reaction torque due to the interaction between PM flux linkage and armature current. 


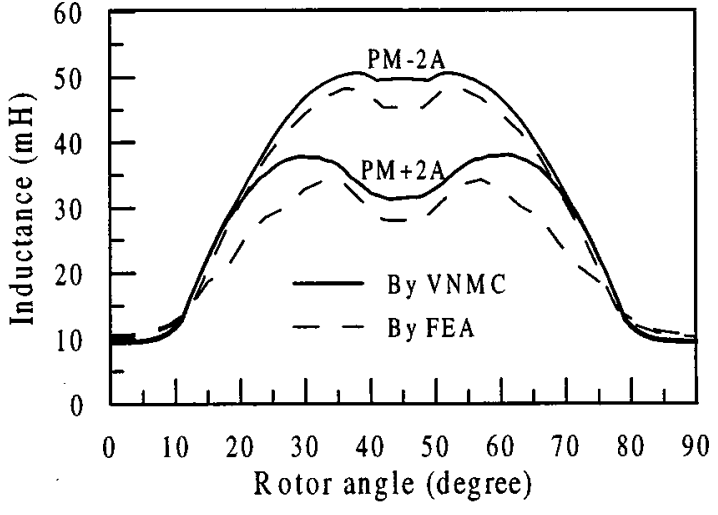

(a)

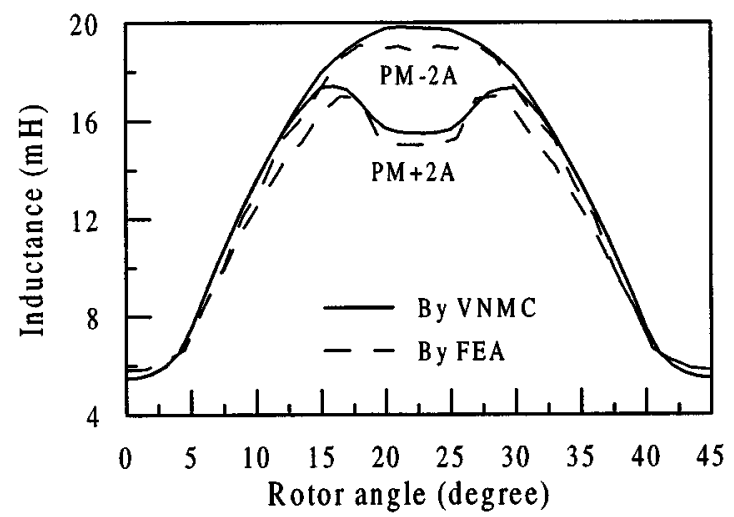

(b)

Fig. 10. Inductances at armature current of 2 A. (a) 6/4-pole. (b) 12/8-pole.

TABLE III

COMPUTATION TIME COMPARISON BETWEEN VNMC AND FEA

\begin{tabular}{c|cc|cc}
\hline \multirow{2}{*}{} & \multicolumn{2}{|c|}{ Time For 6/4-Pole (s) } & \multicolumn{2}{c}{ Time For 12/8-Pole (s) } \\
\cline { 2 - 5 } & PM Flux Linkage & Inductance & PM Flux Linkage & Inductance \\
\hline VNMC & 0.13 & 0.3 & 0.16 & 0.28 \\
FEA & 283 & 355 & 90 & 130 \\
\hline
\end{tabular}

When $i_{a}=2 \mathrm{~A}$, the static torque characteristics of the proposed DSPM motors are illustrated in Fig. 11. It may be seen that $T_{p m}$ is the dominant component of the static torque, while $T_{r}$ has a less significant effect. Thus, the slightly higher discrepancies of those inductances obtained by the VNMC approach will not significantly affect the accuracy of static torque. Finally, the technical data of the two DSPM prototypes are listed in Table IV.

\section{CONCLUSION}

In this paper, a VNMC modeling approach has been developed for the newly invented DSPM motors. Based on the nonlinear VNMC model, the static characteristics of DSPM motors, namely PM flux linkage, inductances, and torque, are calculated, showing that the interaction between PM flux and armature current flux has a significant effect on the inductances. Moreover, correction terms are introduced to the permeance calculations of the airgap and the poles with local saturation, making the calculated static characteristics agree more closely with those found by FEA and measurement. Comparisons also show that the proposed VNMC approach offers the advantage of calculating, effectively and efficiently, the static characteris-

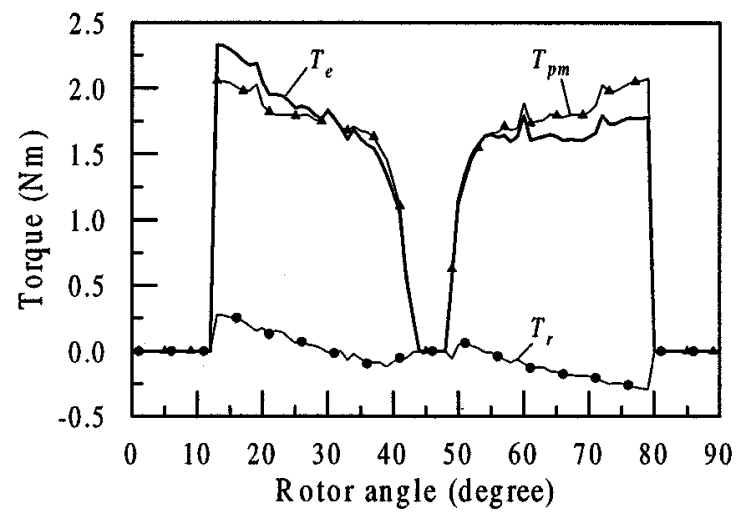

(a)

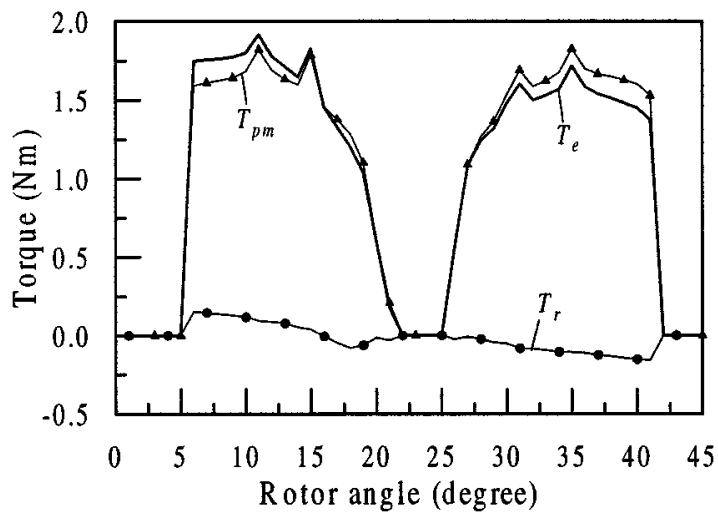

(b)

Fig. 11. Static characteristics of total torque $T_{e}$, PM torque component $T_{p m}$, and reluctance torque component $T_{r}$ : (a) 6/4-pole and (b) 12/8-pole.

TABLE IV

MOTOR TECHNICAL DATA

\begin{tabular}{l|l|l}
\hline Item & $6 / 4-$ pole & $12 / 8-$ pole \\
\hline Rated Power & $750 \mathrm{~W}$ & $750 \mathrm{~W}$ \\
Rated Speed & $1500 \mathrm{rpm}$ & $1500 \mathrm{rpm}$ \\
Number Of Phases & 3 & 3 \\
Stator Inner Diameter & $75 \mathrm{~mm}$ & $75 \mathrm{~mm}$ \\
Airgap Length & $0.45 \mathrm{~mm}$ & $0.45 \mathrm{~mm}$ \\
Stack Length & $75 \mathrm{~mm}$ & $75 \mathrm{~mm}$ \\
Winding Turns Per Phase & 250 & 260 \\
Stator Pole Arc & $30^{\circ}$ & $15^{\circ}$ \\
Stator Pole Height & $15 \mathrm{~mm}$ & $13 \mathrm{~mm}$ \\
Rotor Pole Arc & $36^{\circ}$ & $20^{\circ}$ \\
Rotor Pole Height & $8 \mathrm{~mm}$ & $9 \mathrm{~mm}$ \\
Magnet Dimensions & $75 \times 37.8 \times 6 \mathrm{~mm}$ & $75 \times 18.5 \times 6 \mathrm{~mm}$ \\
Magnet Remanence & $1.08 \mathrm{~T}$ & $1.08 \mathrm{~T}$ \\
Magnet Material & $\mathrm{NdFeB}$ & $\mathrm{NdFeB}$ \\
\hline
\end{tabular}

tics of DSPM motors with different dimensions, parameters, and conditions with reasonable accuracy. Therefore, the proposed approach is especially useful for design optimization of a particular motor type, such as DSPM motors.

\section{REFERENCES}

[1] A. G. Jack, B. C. Mecrow, and J. A. Haylock, "A comparative study of permanent magnet and switched reluctance motors for high-performance fault-tolerant applications," IEEE Trans. Ind. Applicat., vol. 32, no. 4, pp. 889-895, 1996.

[2] C. C. Chan, K. T. Chau, J. Z. Jiang, W. Xia, M. Zhu, and R. Zhang, "Novel permanent magnet motor drives for electric vehicles," IEEE Trans. Ind. Electron., vol. 43, no. 2, pp. 331-339, 1996.

[3] Y. Liao and T. A. Lipo, "A new doubly salient permanent magnet motor for adjustable speed drives," Elect. Machines Power Syst., vol. 22, no. 1, pp. 259-270, 1993. 
[4] Y. Liao, F. Liang, and T. A. Lipo, "A novel permanent magnet motor with doubly salient structure," IEEE Trans. Ind. Applicat., vol. 31, no. 5, pp. 1069-1078, 1995.

[5] M. M. Radulescu, C. Martis, and K. Biro, "A new electronically-commutated doubly-salient permanent-magnet motor," in Proc. Int. Conf. Elect. Machines Drives, Romania, 1995, pp. 213-216.

[6] M. Cheng, K. T. Chau, and C. C. Chan, "A new doubly salient permanent magnet motor," in Proc. IEEE Int. Conf. Power Electron. Drives Energy Syst., Australia, 1998, pp. 2-7.

[7] M. V. K. Chari, G. Bedrosian, J. D' Angelo, and A. Konrad, "Finite element application in electrical engineering," IEEE Trans. Magn., vol. 29, pp. 1306-1314, Mar. 1993.

[8] R. Pohl, "Theory of pulsating-field machines," Proc. Inst. Elect. Eng., pt. II, vol. 93, no. 3, pp. 37-47, 1946.

[9] U. S. Deshpande, J. J. Cathey, and E. Richter, "High-force density linear switched reluctance machine," IEEE Trans. Ind. Applicat., vol. 31, no. 2, pp. 345-352, 1995.

[10] S. H. Khan and A. A. Ivanov, "An analytical method for the calculation of static characteristics of linear step motors for control rod drives in nuclear reactors," IEEE Trans. Magn., vol. 31, pp. 2324-2331, May 1995.

[11] Q. H Zhan, Switch Reluctance Motors. Wuhan, China: Huazhong Univ. Sci. Technol. Press, 1992.

Ming Cheng was born in Jiangsu Province, China. He received the B.Sc.(Eng.) and M.Sc.(Eng.) degrees in electrical engineering in 1982 and 1987, respectively, from Southeast University, Nanjing, China. He is currently working toward the Ph.D. degree at the University of Hong Kong.

Prior to entering the Ph.D. program, he was an Associate Professor with the Department of Electrical Engineering, Southeast University. His areas of interests include electrical machine and drive design, modeling, simulation, PM machines, and power electronics. He has published some 30 papers in this area, and is the recipient or co-recipient of several Chinese patents.
K. T. Chau (M'89) received the first-class honors B.Sc.(Eng.), M.Phil., and $\mathrm{Ph} . \mathrm{D}$. degrees in electrical and electronic engineering from the University of Hong Kong.

Currently, he works as Associate Professor at the University of Hong Kong. His teaching and research interests focus on three main areas-power converters, machines and drives, and electric vehicles. In these areas, he has published over one hundred refereed technical papers and some industrial reports. $\mathrm{He}$ has also served as chairs and organizing committee members for many international conferences. His biography is listed in Who's Who in Science and Engineering 1998-1999.

C. C. Chan (M'77-SM'77-F'92) received the B.Sc. degree from China University of Mining and Technology, Beijing, China, the M.Sc. degree from Tsinghua University, Beijing, China, and the Ph.D. degree from University of Hong Kong, Hong Kong, in 1953, 1957, and 1981, respectively, and was awarded the Honorary D.Sc. degree from the University of Odessa in 1992.

He is currently the Head and Chair Professor of Electrical Engineering in the Department of Electrical and Electronic Engineering, University of Hong Kong. He has authored four books, published over 120 technical papers, and holds seven patents.

Dr. Chan is a Fellow of the Royal Academy of Engineering, U.K., an Academician of the Chinese Academy of Engineering, and a Fellow of the Ukraine Academy of Engineering Science.

E. Zhou graduated in electrical engineering in 1949 from Central University, China. He started his postgraduate study in Moscow Power College, USSR in 1954 and received the M.Sc. degree in 1958.

He is now a Professor in the Department of Electrical Engineering, Southeast University, Nanjing, China. He has authored over 100 technical papers and five books.

X. Huang was born in Yunnan Province, China. He received the B.Sc.(Eng.) and M.Sc.(Eng.) degrees in electrical engineering in 1995 and 1998, respectively, from Southeast University, Nanjing, China.

His research interests include electrical machines, variable speed drives, and power electronics. 